\title{
Intensificación de dosis con radioterapia conformacional 3D en cáncer de próstata. ¿Más dosis es mejor?
}

\author{
A. Zapatero, A. Marín*, A. Cruz-Conde*, M.A. López*, R. Mínguez**, F. García-Vicente*** \\ *Servicios de Oncología Radioterápica. ${ }^{* *}$ Urología y ${ }^{* * *}$ Radiofisica. \\ Hospital Universitario de la Princesa. Madrid
}

Actas Urol Esp 2005; 29 (9): 834-841

\section{RESUME N}

INTENSIFICACIÓN DE DOSIS CON RADIOTERAPIA CONFORMACIONAL 3D EN CÁNCER DE PRÓSTATA. ¿MÁS DOSIS ES MEJOR?

Objetivo: Determinar el efecto de la dosis de radiación en los resultados de control bioquímico y morbilidad en pacientes con cáncer de próstata.

Material y Métodos: Entre 1995 y 2003, 360 pacientes con cáncer de próstata T1-T3b fueron tratados con RTC3D (66,0 Gy-82,6 Gy). Los pacientes fueron asignados a uno de tres grupos pronósticos: a) bajo riesgo: tratados con RTC3D exclusiva; b) riesgo intermedio tratados con deprivación androgénica (DA) neoadyuvante 4-6 meses previos y durante RTC3D; y c) alto riesgo: DA previa, durante y dos años tras RTC3D. Para el análisis de toxicidad tardía se empleó la escala de RTOG/EORTC.

Resultados: Con una mediana de seguimiento de 48 meses (12-138), la supervivencia libre de fracaso bioquímico (SLFB) a 4 años en pacientes de bajo riesgo fue del 88\%, en los de riesgo intermedio fue del $68 \%$ y en los de alto riesgo del 79\%. En el análisis estratificado del efecto de la dosis de RTC3D controlado por el uso de hormonoterapia, y en el análisis multivariante, tanto la dosis alta de irradiación ( $>76$ Gy) ( $\mathrm{p}=0,0053$ ), como el empleo de DA durante dos años en alto riesgo $(\mathrm{p}=0,0046)$ se correlacionan de forma independiente con un mejor control bioquímico. La incidencia de sangrado rectal y urinario grado 2 fue del $7 \%$ y $11 \%$ respectivamente.

Conclusión: Estos datos confirman que tanto la RTC3D a dosis altas como la asociación de DA en pacientes de alto riesgo contribuyen de forma independiente a mejorar de forma significativa los resultados del tratamiento de los pacientes con cáncer de próstata.

Palabras Clave: Cáncer de próstata. Radioterapia conformacional. Escalada de dosis. Deprivación androgénica. Antígeno prostático específico.

\section{ABSTRACT \\ DOSE ESCALATION WITH THREE DIMENSIONAL CONFORMAL RADIOTHERAPY FOR PROSTATE CANCER: MORE IS BETTER?}

Purpose: The present study was undertaken to determine the effect of radiation dose on biochemical control and morbidity in prostate cancer patients.

Materials and Methods: Between 1995 and 2003, 360 patients with T1-T3b prostate cancer were treated in a sequential radiation dose escalation trial from 66.0 to $82.6 \mathrm{~Gy}$. These patients were prospectively assigned to 1 of 3 prognostic groups according to risk factors: a) low risk patients were treated with 3DCRT alone; b) intermediate risk patients were allocated to receive neoadjuvant $\mathrm{AD}$ (NAD) 4-6 months prior and during 3DCRT; and c) highrisk received $\mathrm{NAD}$ and adjuvant $\mathrm{AD}(\mathrm{AAD}) 2$ years after 3DCRT. RTOG/EORTC toxicity score was used to analyze late complications

Results: Median follow-up was 48 months (12-138). The actuarial biochemical disease free survival (bDFS) at 4 years for low risk, intermediate risk and high risk patients was $88 \%, 68 \%$ and $79 \%$ respectively. Stratified and multivariate analysis showed that higher radiation dose ( $>76 \mathrm{~Gy})(\mathrm{p}=0.0053)$ and the use of AAD for high risk patients $(\mathrm{p}=0.0046)$ correlated significantly with an improvement of bDFS for all patients. The incidence of late grade 2 rectal and urinary bleeding were $7 \%$ and $11 \%$ respectively.

Conclusion: The present study confirms an independent benefit of high-dose (> 76 Gy) radiation therapy and long-term $\mathrm{AAD}$ in high-risk prostate cancer patients.

Keywords: Prostate cancer. Conformal radiotherapy. Dose escalation. Androgen deprivation. Prostate-specific antigen. 
$\mathrm{E}$ n las últimas dos décadas, en el ámbito de la radioterapia, han emergido dos conceptos encaminados a mejorar el control local y supervivencia de los pacientes con cáncer de próstata localizado, fundamentalmente la intensificación de dosis de radiación con nueva tecnología de radioterapia conformacional tridimensional (RTC3D) y el tratamiento combinado hormonoradioterapia.

Los resultados de múltiples estudios monoinstitucionales y multi-institucionales de escalada de dosis con RTC3D han demostrado de forma consistente una mejoría en el control bioquímico y control local cuando se elevan las dosis de radiación ${ }^{1-6}$. Este beneficio que fue inicialmente probado para pacientes con enfermedad de riesgo intermedio y alto riesgo, en estudios recientes se extiende también a pacientes con enfermedad precoz de bajo riesgo ${ }^{7,8}$.

En la actualidad existe abundante evidencia científica publicada, que sostiene el uso combinado de DA y RT en pacientes seleccionados con cáncer de próstata, particularmente aquéllos con enfermedad localmente avanzada y/o factores de alto riesgo ${ }^{9-14}$. Sin embargo, debido a que los ensayos aleatorizados publicados que han demostrado un beneficio en supervivencia con la combinación de DA y RT han empleado esquemas y dosis de radioterapia convencionales $(\leq 70$ Gy), queda por definir cual es el efecto clínico de la interacción entre dosis altas de radiación y la DA.

En este estudio, los autores analizan el beneficio potencial de la dosis alta de radiación en los resultados de control bioquímico de pacientes con cáncer de próstata localizado tratados con RTC3D dentro de un estudio prospectivo, y de forma secundaria el impacto adicional de la DA en pacientes seleccionados de riesgo elevado.

\section{PACIENTES Y MÉTODOS}

Características de los pacientes

Entre 1995 y 2003, 360 pacientes con cáncer de próstata T1-T3b NO MO fueron tratados con RTC3D dentro de un protocolo institucional de escalada de dosis desde 66,0 Gy a 82,6 Gy. Todos los pacientes fueron prospectivamente asignados a uno de tres grupos de tratamiento en relación con factores pronósticos (nivel de PSA al diagnós- tico, grado de Gleason y estadio T). Ochenta y un pacientes de riesgo intermedio (presencia de un factor desfavorable: grado de Gleason $\geq 8$, estadio T3 o PSA 220 ) recibieron DAN 4-6 meses previos y durante RT. Ciento treinta y siete pacientes de alto riesgo (más de un factor desfavorable) recibieron DAA prolongada (previa, durante y dos años tras RT). Los restantes 142 pacientes con criterios de bajo riesgo (ningún factor desfavorable) se trataron con RT exclusiva.

La mediana de los valores de estos pacientes es: edad 70 años (rango 50-79); PSA pre-tratamiento $12,8 \mathrm{ng} / \mathrm{ml}$ (rango 2,8-741); y dosis RT ICRU 74,0 Gy (rango 66,0-82,6 Gy). La distribución de pacientes por estadios según la American Join Committee on Cancer (AJCC) de 1997 fue T1: 78 (22\%), T2: 145 (40\%) y T3: 137 (38\%). El grado de Gleason fue disponible en todos los pacientes: Gleason $\leq 6$ en $59 \%$ de los pacientes, Gleason 7 en $25 \%$ y Gleason 8-10 en 16\%. Las características de los pacientes quedan resumidas en la Tabla 1.

\section{Tratamiento Radioterápico}

Las técnicas de simulación y planificación terapéutica han sido descritas con anterioridad $^{1,2}$. Brevemente, todos los pacientes fueron sometidos a realización de tomografía computarizada (TC) (con cortes cada 0,5-0,3 cm) en condiciones anatómicas de tratamiento: posición decúbito supino empleando un sistema individualizado de inmovilización para pies y rodillas, con el recto vacío y la vejiga llena. El volumen de tratamiento (gross tumor volume: GTV y clinical target volume: CTV) consistió en la glándula prostática con o sin vesículas seminales, dependiendo del riesgo estimado de afectación de vesículas seminales (basado en datos del grado de Gleason, PSA al diagnóstico y estadio T), utilizándose para su localización las imágenes de TC realizado con uretrograma retrógrado. Desde 1995 hasta diciembre 1999, el CTV incluyó también de forma profiláctica los ganglios pélvicos en aquellos pacientes de alto riesgo, hasta una dosis de 45,050,0 Gy. Todos los pacientes se trataron con fotones de $25 \mathrm{MV}$.

La escalada de dosis se inició con 66,0 Gy y fue incrementándose sucesivamente y de forma controlada hasta 82,6 Gy. En general en los 
Tabla 1

Características de los pacientes

\begin{tabular}{|c|c|}
\hline $\mathrm{N}$ & 360 \\
\hline $\begin{array}{l}\text { EDAD } \\
\text { años (mediana, media, rango) } \\
\leq 65 \text { años } \\
>65 \text { años }\end{array}$ & $\begin{array}{l}70,68,5(50-79) \\
94(26 \%) \\
266(74 \%)\end{array}$ \\
\hline $\begin{array}{c}\text { ESTADIO T } \\
\text { T1 } \\
\text { T2 } \\
\text { T3 }\end{array}$ & $\begin{array}{r}78(22 \%) \\
145(40 \%) \\
137(38 \%)\end{array}$ \\
\hline $\begin{array}{l}\text { PSA } \\
\text { (ng/ml): media; mediana (rango) } \\
\quad \leq 10 \\
\quad 10-20 \\
\quad>20\end{array}$ & $\begin{array}{l}26.13 ; 12.8 ;(2.8-741) \\
133(36 \%) \\
125(35 \%) \\
102(29 \%)\end{array}$ \\
\hline $\begin{array}{l}\text { GLEASON } \\
\quad \leq 6 \\
\quad 7 \\
\quad \geq 8\end{array}$ & $\begin{array}{l}212(59 \%) \\
91(25 \%) \\
57(16 \%)\end{array}$ \\
\hline $\begin{array}{l}\text { HORMONOTERAPIA } \\
\text { Inducción } \\
\text { Inducción+Mantenimiento } \\
\text { Ninguna }\end{array}$ & $\begin{array}{l}81(23 \%) \\
137(38 \%) \\
142(39 \%)\end{array}$ \\
\hline $\begin{array}{l}\text { DOSIS ICRU } \\
\text { mediana; (rango) } \\
\quad \leq 70 \mathrm{Gy:} \\
\quad>70 \mathrm{~Gy}-\leq 76 \mathrm{~Gy} \\
>76 \mathrm{~Gy}\end{array}$ & $\begin{array}{l}74 \text { Gy }(66,0 \text { Gy-82,6 Gy) } \\
123(34 \%) \\
90(26 \%) \\
141(40 \%)\end{array}$ \\
\hline $\begin{array}{l}\text { RECAIDA PSA } \\
\text { Sí } \\
\text { No }\end{array}$ & $\begin{array}{l}86(24 \%) \\
274 \quad(76 \%)\end{array}$ \\
\hline $\begin{array}{l}\text { TIEMPO RECAÍDA } \\
\text { media/mediana, rango }\end{array}$ & $45 \mathrm{~m} / 43 \mathrm{~m}(9-132 \mathrm{~m})$ \\
\hline BIOPSIAS HECHAS & $111(33 \%)$ \\
\hline BIOPSIAS POSITIVAS & $27 / 111 \quad(24 \%)$ \\
\hline METÁSTASIS & $10 / 360 \quad(2.8 \%)$ \\
\hline VIVOS & $357 / 360 \quad$ (99\%) \\
\hline $\begin{array}{l}\text { VIVOS SIN ENFERMEDAD } \\
\text { VISIBLE }\end{array}$ & $339 / 360 \quad$ (94\%) \\
\hline
\end{tabular}

pacientes tratados con dosis $\leq 72,0$ Gy se utilizó la técnica de cuatro campos, y en los que recibieron dosis $\geq 75,6$ Gy se empleó la técnica de 6 campos en estrella (dos laterales opuestos y cuatro oblicuos). Los resultados se analizaron registrando la dosis ICRU [International Commission Radiation Units and Measurement] de referencia en el isocentro y categorizándola en 3 niveles de dosis: 1) $\leq 70,0$ Gy en 123 (34\%) pacientes, 2) $>70,0$ Gy y $\leq 76$ Gy en 90 (26\%) pacientes, y 3) > 76 Gy en 141 (40\%) pacientes.

\section{Deprivación Androgénica}

El tratamiento hormonal se administró a pacientes de riesgo como parte de un estudio prospectivo multi-institucional ${ }^{15-17}$. Ochenta y un pacientes de riesgo intermedio recibieron DAN 46 meses previos y durante RT y 137 pacientes de alto riesgo recibieron DAA prolongada (previa, durante y dos años tras RT). En la mayoría (95\%) de los pacientes la supresión androgénica consistió en la combinación de un análogo de LHRH y un antiandrógeno no esteroideo.

\section{Toxicidad tardía}

Para el análisis de toxicidad tardía se empleó la escala de la RTOG/EORTC modificada para sangrado rectal ${ }^{18}$. Brevemente y entre otros, se considera morbilidad grado 2 la presencia de sangrado rectal o hematuria intermitente, y grado 3 aquéllos sangrados que por su importancia requieran transfusión de concentrados de hematíes, cauterización o lavados.

\section{Seguimiento}

Las visitas de seguimiento (que incluyeron tacto rectal y analítica con determinación de PSA $\mathrm{y}$ testosterona) fueron realizadas con una periodicidad de 3-4 meses en la primera revisión, cada seis meses, después durante 5 años y posteriormente con frecuencia anual. En los pacientes con criterios de fracaso bioquímico se realizó además, TC abdomino-pélvico, radiografía de tórax, gammagrafía ósea y biopsia prostática. A los pacientes que recibieron tratamiento hormonal durante dos años también se les realizó este estudio al finalizar el tratamiento hormonal. La mediana de seguimiento es de 48 meses (rango 12-138).

\section{Análisis estadístico}

El objetivo primario del estudio fue la supervivencia libre de fracaso bioquímico (SLFB), que fue calculada siguiendo la definición consenso de the American Society for Therapeutic Radiology and Oncology (ASTRO 1997) ${ }^{19}$ pero señalando el evento en nuestro caso, en la fecha de tercer ascenso consecutivo de PSA. La SLFB se calculó desde la 
fecha del diagnóstico hasta el evento o el último seguimiento, mediante el método Kaplan-Meier. Las diferencias entre grupos se compararon mediante el log-rank test para variables dicotómicas y mediante la comparación de medias para variables continuas. Se definió supervivencia libre de enfermedad (SLE) como la presencia de cualquier fracaso clínico visible en imagen o palpable, y fue calculado desde la fecha del diagnóstico hasta el evento. Con el fin de determinar el efecto de la dosis de RT en la SLFB, la dosis ICRU se analizó tanto como variable continua, como categorizada en 3 subgrupos. Se realizó un análisis univariante y posteriormente multivariante (regresión de Cox), con el objetivo de determinar el valor pronóstico independiente de variables clínicas de interés (edad, PSA al diagnóstico, grado de Gleason, estadio clínico T) y variables terapéuticas (dosis de radiación y empleo de hormonoterapia). Se consideró un nivel de significación $\mathrm{P}<0,05$ (two-sided) en todos los tests estadísticos. El análisis se realizó usando el software SPSS v. 10,0 .

\section{RESULTADOS}

Control bioquímico

De los 360 pacientes, ochenta y seis (24\%) desarrollaron fracaso bioquímico como primer evento, con un tiempo mediano hasta la recaída de 43 meses (rango 9-132 meses). El 50\% de los pacientes experimentaron la recaída en los primeros 43 meses y el $75 \%$ antes de los cinco años (56 meses). Dentro de un sub-estudio institucional, se realizaron biopsias prostáticas en 111 pacientes (33\%) en dos situaciones: como control a los 24-36 meses del tratamiento radioterápico y biopsias diagnósticas en pacientes con fracaso bioquímico. Se encontraron hallazgos compatibles con persistencia de carcinoma prostático en 27 de los 111 pacientes biopsiados (24\%). De estos 27 pacientes con biopsias positivas, $20(74 \%)$ tenían fracaso bioquímico previo $(\mathrm{p}<0,0001)$. Se demostró metástasis a distancia en $10(2,8 \%)$ pacientes con una mediana de tiempo a su aparición de 17 meses (rango 12-40).

De los 360 pacientes, 357 (99\%) se encuentran vivos y 339 (94\%) vivos y libres de enfermedad visible/palpable. La SLFB actuarial a 4 años para los 360 pacientes es del 78\% (error estándar
[EE]:0,0266). En los pacientes de bajo riesgo la SLFB fue del 88\% (EE: 0,0472), en los de riesgo intermedio la SLFB fue del 68\% (EE: 0,0636) y en los de alto riesgo la SLFB fue del 79\% (EE: 0,0352).

\section{Análisis de factores pronósticos}

Los resultados del análisis univariante muestran que cuando los pacientes reciben un tratamiento protocolizado acorde a sus factores de riesgo (RT exclusiva, o combinada con DA corta o mantenida), no hay diferencias significativas en el control bioquímico de la enfermedad ni en relación con la edad del paciente $(\mathrm{p}=0,1919)$, ni con el estadio $\mathrm{T}(\mathrm{p}=0,1978)$, ni con el nivel de PSA al diagnóstico $(p=0,1011)$. Sólo el grado de Gleason 8-10 se asoció con un peor resultado en control bioquímico ( $p<0,0187)$. La SLFB a 4 años fue del $71 \%$ en pacientes con grado de Gleason 8-10, frente a $86 \%$ en Gleason 7 y $77 \%$ en Gleason $<7$.

En cuanto al objetivo primario del estudio, los resultados de este análisis indican que la dosis alta de irradiación (tanto como variable continua como variable categórica) se asocia de forma muy significativa a un beneficio en control bioquímico. La SLFB estimada a 4 años en pacientes tratados con dosis RT $>76$ Gy fue del $94 \%$ comparada con $82 \%$ para dosis entre 70 y 76 Gy, y $65 \%$ para dosis $\leq 70$ Gy $(p<0,00001)$. Este beneficio de dosis elevadas de irradiación es evidente en los tres grupos de riesgo (bajo, intermedio y alto) (Fig. 1). En este análisis, el empleo de hormonoterapia prolongada durante dos años se correlacionó también de forma significativa con una mejoría en el control bioquímico. La SLFB a 4 años para los pacientes tratados con DAA fue del 95\% frente a un $71 \%$ en los que no la recibieron $(\mathrm{p}=0,0001)$. Los resultados del análisis estratificado valorando el efecto de la dosis de RTC-3D en la SLFB ajustada o controlada por el empleo o no de DA de mantenimiento fueron también estadísticamente significativos $(\mathrm{p}=0,0004)$, indicando el valor pronóstico independiente de la dosis de irradiación y la hormonoterapia de mantenimiento (Tabla 2) (Fig. 2).

Posteriormente se realizó un análisis multivariante de toda la serie, incluyendo todas las variables significativas identificadas en el análisis univariante (Tabla 3). Los resultados confirman 


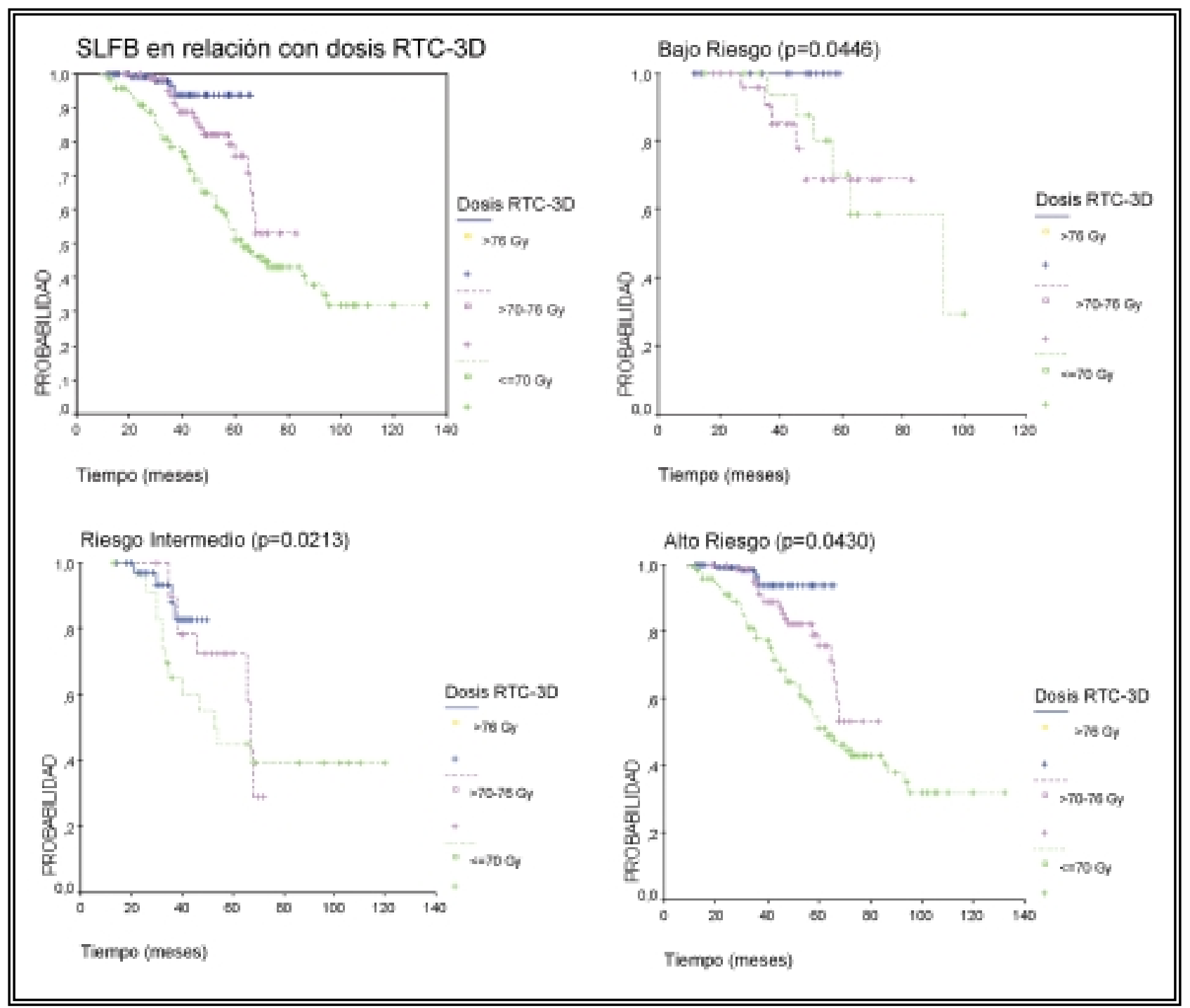

FIGURA 1. SLFB global en relación con dosis RTC-3D $(n=360)(p=0,0053)$ y en los subgrupos de bajo (p=0.0446), intermedio $(p=0,0213)$ y alto riesgo $(p=0,0430)$.

que tanto las dosis altas de irradiación (>76 Gy punto ICRU) ( $\mathrm{p}=0,0053)$, el empleo de hormonoterapia durante dos años $(\mathrm{p}=0,0046)$ y el grado de Gleason $<8$ ( $\mathrm{p}=0,0111)$, se correlacionan de forma independiente y significativa con un mejor pronóstico en términos de control bioquímico.

\section{Morbilidad Tardía}

Todos los pacientes $(\mathrm{N}=360)$ fueron analizables para complicaciones tardías, y 331 pacientes fueron valorables para el análisis de sangrado rectal y hematuria (Tabla 4). La incidencia de complicaciones intestinales grado $\geq 2$ fue del $10 \%$ ( $9 \%$ grado 2 y $1 \%$ grado 3). Entre ellas se objetivó sangrado rectal grado 2 en 24 casos (7\%) y grado 3 en 2 casos (0,6\%). La incidencia de complicaciones urinarias grado $\geq 2$ fue del $15 \%$ (14\% grado 2 y $1 \%$ grado 3$)$. De estas complicaciones la más frecuentemente observada fue la hematuria que resultó de grado 2 en 35 casos (11\%) y de grado 3 en 2 casos $(0,6 \%)$. El resto fueron pacientes con aumento de la frecuencia miccional diurna o nocturna. No hubo ningún caso de toxicidad grado $\geq 4$.

\section{DISCUSIÓN}

La dosis de radiación representa hoy en día un factor importante en el control tumoral con radioterapia del cáncer de próstata. Con el beneficio de la intensificación de dosis con radioterapia con- 
Tabla 2

SLFB en relación a dosis de RTC3D ajustada (estratificada) por empleo de hormonoterapia de mantenimiento.

\begin{tabular}{lcccc}
\hline & N & Eventos & Censores & (\%) \\
\hline Da. manten./si & 131 & 16 & 115 & 87,79 \\
Dosis $\leq 70$ Gy & 27 & 10 & 17 & 62,96 \\
Dosis <70-76 Gy & 42 & 5 & 37 & 88,10 \\
Dosis > 76 Gy & 62 & 1 & 61 & 98,39 \\
& & & & \\
Da. manten./no & 221 & 70 & 151 & 68,33 \\
Dosis $\leq 70$ Gy & 96 & 52 & 44 & 45,83 \\
Dosis <70-76 Gy & 47 & 14 & 33 & 70,21 \\
Dosis > 76 Gy & 78 & 4 & 74 & 94,87 \\
Global & 352 & 86 & 266 & 75,57 \\
\hline
\end{tabular}

Significación estadística: $\mathrm{p}=0,0004$

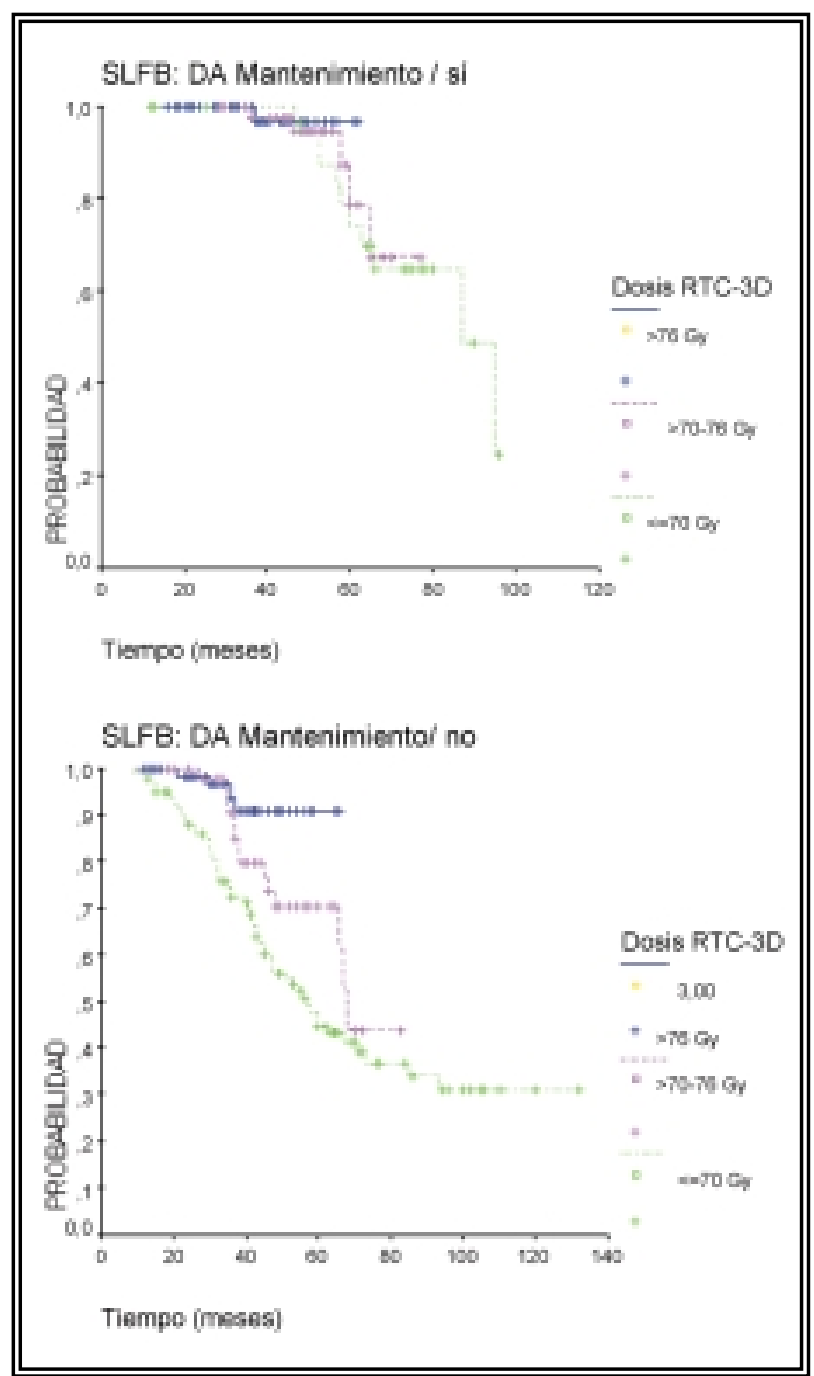

FIGURA 2. SLFB en relación con la dosis de RTC-3D estratificada o ajustada por el empleo de deprivación androgénica (DA) de mantenimiento ( $p=0004)$.
Tabla 3

Análisis Multivariante

\begin{tabular}{lcccc}
\hline Variable & Beta & HR & P & IC95\% \\
\hline Da. mant. & $-0,9513$ & 0,3862 & 0,0046 & $0,1999-0.7462$ \\
& & & & \\
Gleason & & & 0,0111 & \\
$\quad>$ 7 VS $<7$ & $-0,8410$ & 0,4313 & 0,0060 & $0,2367-0.7857$ \\
$>$ 7 VS 7 & $-0,9092$ & 0,4028 & 0,0087 & $0,2043-0.7943$ \\
& & & & \\
Dosis RT Gy & & & 0,0053 & \\
$\quad>76$ VS <70 & 1,5012 & 4,4870 & 0,0017 & $1,7575-11.4556$ \\
$>$ 76 VS 70-76 & 1,1090 & 3,0313 & 0,0290 & $1,1204-8.2016$ \\
\hline
\end{tabular}

HR: hazard ratio; IC: intervalo de confianza: DA: deprivación androgénica

formacional, asistimos a una creciente controversia sobre el papel de la supresión androgénica en asociación con altas dosis de radioterapia, o la necesidad de escalar dosis de RT cuando se emplea tratamiento hormonal en pacientes con cáncer de próstata de riesgo alto. Conforme los datos de la literatura se acumulan, la cuestión crítica por resolver es si la combinación de ambas modalidades, radioterapia conformada en intensificación de dosis y la supresión androgénica, puede mejorar los resultados de cada una en particular.

En este trabajo se presenta la experiencia del Hospital Universitario de la Princesa en cáncer de próstata con RTC-3D durante 10 años. El objetivo de este estudio preliminar es evaluar los resultados de control bioquímico del programa de RTC3D en escalada de dosis en pacientes con cáncer de próstata localizado, y de forma secundaria analizar el impacto adicional de su asociación con DA prolongada en pacientes de alto riesgo.

Los resultados de SLFB a 4 años del $78 \%$ en toda la serie y en particular en pacientes de bajo riesgo $(88 \%)$ y riesgo intermedio (68\%) están en consonancia con los de otras series publicadas de radioterapia a dosis altas ${ }^{1-2,6,8}$. Los resultados en pacientes de alto riesgo (SLFB a 4 años del 79\%) con la combinación de radioterapia a dosis altas y DA prolongada, aunque preliminares, son muy prometedores cuando se comparan con series de hormonoterapia y radioterapia convencional ${ }^{10,14}$.

Como se esperaba, los resultados tanto del análisis univariante $(\mathrm{p}<0,0001)$ como multivariante $(\mathrm{p}=0,0053)$ confirman que el empleo de RTC-3D a dosis altas en cáncer de próstata, se asocia de forma independiente con un beneficio significativo 
Tabla 4

Complicaciones tardías según escala de toxicidad RTOG/EORTC (n=331)

\begin{tabular}{|c|c|c|c|c|c|}
\hline \multirow[b]{2}{*}{ Tipo } & \multicolumn{5}{|c|}{ Grado } \\
\hline & $\mathbf{0}$ & 1 & 2 & 3 & 4 \\
\hline Urinarias & 198 (55\%) & $109(30,4 \%)$ & 52 (14\%) & $2(0,6 \%)$ & 0 \\
\hline Hematuria & 266 (80\%) & $28(8,5 \%)$ & $35(10,6 \%)$ & $2(0,6 \%)$ & 0 \\
\hline Intestinales & $243(67,5 \%)$ & $81(22,5 \%)$ & $31(8,6 \%)$ & $5(1,4 \%)$ & 0 \\
\hline Sangrado rectal & 267 (80,6\%) & 38 (11,5\%) & $24(7,3 \%)$ & $2(0,6 \%)$ & 0 \\
\hline
\end{tabular}

en el control bioquímico. La SLFB a 4 años en los pacientes tratados con RT a dosis $>76$ Gy fue del 94\% comparada con $82 \%$ para dosis entre $70-76$ Gy, y 65\% para dosis $\leq 70$ Gy. Estos resultados son superponibles a los datos de intensificación de dosis extensamente publicados por otros centros, confirmando la necesidad de administrar dosis altas de radiación para conseguir el máximo control en el cáncer de próstata.

Lo más interesante, es que esta mejoría significativa de los resultados con dosis altas de radiación fue incluso evidente en pacientes de alto riesgo tratados con DA mantenida durante 2 años. Los resultados del análisis multivariante señalan que este beneficio de dosis altas frente a dosis convencionales de radiación es independiente del empleo de hormonoterapia prolongada (HR: 4,48; $\mathrm{p}=0,0053$ ), y parecen confirmar que el tratamiento con DA en pacientes de alto riesgo no sustituye a las dosis altas de radiación.

Este trabajo ha analizado también el impacto sobre el control bioquímico del empleo de DA prolongada durante dos años en combinación con radioterapia. Con una mediana de seguimiento de 48 meses, los resultados indican que la adición de DAA durante 2 años en pacientes de alto riesgo se asocian con un beneficio significativo en el control bioquímico y con independencia de la dosis de radiación (HR: 0,38; $\mathrm{p}=0,0046$ ). Estos datos son consistentes con importantes ensayos aleatorizados que han demostrado una mejoría en supervivencia libre de progresión y supervivencia global en pacientes con cáncer de próstata de riesgo elevado tratados con hormonoterapia $^{10,12,14,20}$. Sin embargo, en todos estos ensayos la dosis de radiación estaba limitada a $\leq 70$ Gy. En la actualidad no existen resultados publica- dos de ensayos fase III con la combinación de hormonoterapia y radioterapia a dosis altas. Los resultados de SLFB a 4 años del 79\% del subgrupo desfavorable de esta serie son alentadores y sugieren un potencial efecto sinérgico entre la radioterapia a dosis altas y la supresión androgénica.

Los autores reconocen las limitaciones inherentes de los estudios no aleatorizados, en particular las relacionadas con el empleo de tratamiento hormonal, ya que este tratamiento confiere una ventaja transitoria a los pacientes tratados con DAA. Por otro lado, la aplicabilidad de los criterios de fracaso de la ASTRO a estos pacientes puede contribuir a cierta confusión en los resultados. Sin embargo, los resultados del análisis multivariante son inequívocos y confirman que tanto la dosis alta de radiación en todos los pacientes, como el empleo de hormonoterapia prolongada en las indicaciones aplicadas en este estudio (pacientes de alto riesgo), contribuyen de forma independiente a mejorar de forma significativa los resultados del tratamiento de los pacientes con cáncer de próstata. Sin embargo, son necesarios los resultados de estudios aleatorizados ya en marcha para confirmar un potencial efecto sinérgico entre ambas modalidades.

En este trabajo, se ha revisado también la incidencia de complicaciones tardías derivadas del tratamiento, específicamente urinarias e intestinales. Los resultados de toxicidad tardía rectal han sido ya previamente publicados ${ }^{16}$. En concreto, la incidencia de toxicidad rectal grado $2(9 \%)$ y específicamente sangrado rectal grado $2(7 \%)$ y grado 3 del $(0,6 \%)$ de esta serie, son comparables de forma favorable con otras series internacionales de radioterapia a dosis altas ${ }^{21-23}$. En cuanto a complicaciones urinarias grado $2 \mathrm{su}$ frecuencia $(15 \%)$ es similar también a otras series $^{24}$. Es importante destacar la escasa frecuencia de complicaciones grado $3(\leq 1 \%)$ de cualquier tipo, y la ausencia de complicaciones grado 4-5. Estos datos confirman que la RTC-3D a dosis altas en pacientes con cáncer de próstata es una técnica de tratamiento segura y efectiva que se asocia a una baja incidencia de toxicidad tardía. 
Agradecimientos: Este trabajo se ha realizado bajo la supervisión científica del GICOR (Grupo de Investigación Oncológica Radioterápica). Los autores agradecen a Francisco Rodríguez, de la Fundación Biomédica del Hospital de la Princesa su supervisión en el análisis estadístico.

\section{REFERENCIAS}

1. Pollack A, Zagars GK, Starkschall G, Antolak JA, Lee JJ, Huang E, et al. Prostate cancer radiation dose response: results of the M. D. Anderson phase III randomized trial. Int J Radiat Oncol Biol Phys 2002;53:1097-1105.

2. Zelefsky MJ, Leibel SA, Gaudin PB, Kutcher GJ, Fleshner NE, Venkatramen ES, et al. Dose escalation with threedimensional conformal radiation therapy affects the outcome in prostate cancer. Int J Radiat Oncol Biol Phys 1998; 41:491-500

3. Zelefsky MJ, Fuks Z, Hunt M, Lee HJ, Lombardi D, Ling CC, et al. SA. High dose radiation delivered by intensity modulated conformal radiotherapy improves the outcome of localized prostate cancer. J Urol 2001;166:876-881.

4. Fiveash JB, Hanks G, Roach M, Wang S, Vigneault E, McLaughlin PW, et al. 3D conformal radiation therapy (3DCRT) for high grade prostate cancer: a multi-institutional review. Int $\mathrm{J}$ Radiat Oncol Biol Phys 2000;47:335-342.

5. Vicini FA, Abner A, Baglan KL, Kestin LL, Martinez AA. Defining a dose-response relationship with radiotherapy for prostate cancer: is more really better? Int $\mathrm{J}$ Radiat Oncol Biol Phys 2001;51:1200-1208.

6. Hanks GE, Hanlon AL, Epstein B, Horwitz EM. Dose response in prostate cancer with 8-12 years' follow-up. Int J Radiat Oncol Biol Phys 2002;54:427-435.

7. Zietman AL, DeSilvio M, Slater JD et al: A randomized trial comparing conventional dose (70.2GyE) and high-dose (79.2GyE) conformal radiation in early stage adenocarcinoma of the prostate: Results of an interim analysis of PROG 95-09 (Abstr.). Int J Radiat Oncol Biol Phys 2004;60:131.

8. P.A. Kupelian, D. Kuban, H. Thames, L. Levy, E. Horwitz, A. Martinez, et al. Improved biochemical relapse-free survival with increased external radiation doses in patients with localized prostate cancer: the combined experience of nine institutions in patients treated in 1994 and 1995 (Abstr.). Int J Radiat Oncol Biol Phys. 2003;57(2Suppl): S271-S272.

9. Roach M 3RD, Lu J, Pilepich MV, Asbell SO, Mohiuddin M, Terry R, et al. Predicting long-term survival, and the need for hormonal therapy: a meta-analysis of RTOG prostate cancer trials. Int J Radiat Oncol Biol Phys. 2000;47:617627.

10. Bolla M, Collette L, Blank L, Warde P, Dubois JB, Mirimanoff $\mathrm{RO}$, et al. Long-term results with immediate androgen suppression and external irradiation in patients with locally advanced prostate cancer (an EORTC study): a phase III randomized trial. Lancet. 2002;360:103-106.

11. Pilepich MV, Winter K, John MJ, Mesic JB, Sause W, Rubin $\mathrm{P}$, et al. Phase III radiation therapy oncology group (RTOG) trial 86-10 of androgen deprivation adjuvant to definitive radiotherapy in locally advanced carcinoma of the prostate. Int J Radiat Oncol Biol Phys.2001;50:1243-1252.

12. Lawton CA, Winter K, Murray K, Machtay M, Mesic JB, Hanks GE, et al. Updated results of the phase III Radiation Therapy Oncology Group (RTOG) trial 85-31 evaluating the potential benefit of androgen suppression following standard radiation therapy for unfavorable prognosis carcinoma of the prostate. Int J Radiat Oncol Biol Phys. 2001;49:937946.
13. Laverdiere J, Gomez JL, Cusan L, Suburu ER, Diamond P, Lemay M, et al. Beneficial effect of combination hormonal therapy administered prior and following external beam radiation therapy in localized prostate cancer. Int $\mathrm{J}$ Radiat Oncol Biol Phys. 1997;37:247-252.

14. Hanks GE, Pajak TF, Porter A, Grignon D, Brereton H, Venkatesan V, et al. Radiation Therapy Oncology GroupPhase III trial of long-term adjuvant androgen deprivation after neoadjuvant hormonal cytoreduction and radiotherapy in locally advanced carcinoma of the prostate: the Radiation Therapy Oncology Group Protocol 92-02. J Clin Oncol. 2003; 21:3972-3978.

15. Zapatero A, Cruz-Conde A, Mínguez R, Rodríguez F, PérezTorrubia A. Control Bioquímico tras Radioterapia Externa en el Cáncer de Próstata Localizado: Resultados de una Cohorte Moderna. Act Urol Esp 2000;24(1):10-18.

16. Zapatero A, García-Vicente F, Modolell I, Alcántara P, Floriano A, Cruz-Conde A, et al. Impact of mean rectal dose on late rectal bleeding following conformal radiotherapy for prostate cancer: dose volume effect. Int J Radiat Oncol Biol Phys 2004;59(5):1343-1351.

17. Zapatero A, Valcárcel F, Calvo FA, Algás R, Béjar A, Maldonado $\mathrm{J}$, et al. Risk-adapted androgen deprivation and escalated 3D-conformal radiotherapy for prostate cancer: does radiation dose influence outcome of patients treated with adjuvant androgen deprivation? Report of a multicenter trial (GICOR 05/99). J Clin Oncol 2005;23:65616568.

18. Lawton CA, Won M, Pilepich MV, Asbell SO, Shipley WU, Hanks GE, et al. Long-term treatment sequelae following external beam irradiation for adenocarcinoma of the prostate: analysis of RTOG studies 7506 and 7706.Int J Radiat Oncol Biol Phys. 1991;21(4):935-939.

19. American Society for Therapeutic Radiology and Oncology Consensus Panel. Consensus statement: Guidelines for PSA following radiation therapy. Int $\mathrm{J}$ Radiat Oncol Biol Phys. 1997;37:1035-1041.

20. Pilepich MV, Winter K, Lawton CA et al. Androgen suppression adjuvant to definitive radiotherapy in prostate carcinoma. Long-term results of phase III RTOG 8531. Int J Radiat Oncol Biol Phys, 2005;61:1285-1290.

21. Skwarchuk MW, Jackson A, Zelefsky MJ, Venkatraman ES, Cowen DM, Levegrun S, et al. Late rectal toxicity after conformal radiotherapy of prostate cancer (I): multivariate analysis and dose-response. Int $\mathrm{J}$ Radiat Oncol Biol Phys. 2000;47(1): 103-113.

22. Fiorino C, Cozzarini C, Vavassori V, Sanguineti G, Bianchi C, Cattaneo GM, et al. Relationships between DVHs and late rectal bleeding after radiotherapy for prostate cancer: analysis of a large group of patients pooled from three institutions. Radiother Oncol. 2002 Jul;64(1):1-12.

23. Huang EH, Pollack A, Levy L, Starkschall G, Dong L, Rosen I, et al. Late rectal toxicity: dose-volume effects of conformal radiotherapy for prostate cancer. Int $\mathrm{J}$ Radiat Oncol Biol Phys. 2002 Dec 1;54(5):1314-1321.

24. Michalski JM, Winter K, Purdy JA, Wilder RB, Perez CA, Roach M, et al. Preliminary evaluation of low-grade toxicity with conformal radiation therapy for prostate cancer on RTOG 9406 dose levels I and II. Int J Radiat Oncol Biol Phys. 2003 May 1;56(1):192-198.

Dra. A. Zapatero

Servicio de Oncología Radioterápica.

Hospital Universitario de la Princesa

Diego de León, 62 - 28006 Madrid

e-mail: hlpr@salud.madrid.org

(Trabajo recibido el 11 de julio 2005) 